\title{
VALORES REFERENCIAIS DA ELETROMIOGRAFIA DE MÚSCULOS ENVOLVIDOS NA DEGLUTIÇÃO: UMA REVISÃO SISTEMÁTICA
}

\section{Reference values for the electromyography of muscles involved in swallowing: a systematic review}

\author{
Luciana Rodrigues Belo ${ }^{(1)}$, Maria das Graças Wanderley de Sales Coriolano (2), \\ Danielle Carneiro de Menezes ${ }^{(3)}$, Otávio Gomes Lins ${ }^{(4)}$
}

\begin{abstract}
RESUMO
Tema: normalidade da eletromiografia de superfície de músculos envolvidos na deglutição. Objetivo: investigar se a literatura aponta valores referenciais de normalidade para a duração, amplitude e características dos eletromiogramas dos músculos envolvidos na deglutição (orbicular da boca, masseter, músculos da região supra-hióidea e músculos da região infra-hióidea cobertos pelo músculo platisma). Conclusão: a busca resultou em 33 referências, das quais somente cinco enquadraram-se nos critérios de inclusão. Apenas uma referência foi classificada com um bom nível de qualidade pela escala de Jadad com modificações. Os artigos selecionados podem não apontar valores referenciais confiáveis principalmente para a amplitude e morfologia da eletromiografia de superfície, pois utilizaram uma freqüência de amostragem inadequada para os registros eletromiográficos o que potencializa a obtenção de dados distorcidos da atividade muscular. Tendo em vista a variabilidade inter e entre os sujeitos, a literatura sugere a realização de técnicas de normalização do sinal eletromiográfico.
\end{abstract}

DESCRITORES: Eletromiografia; Deglutição; Músculos

\section{INTRODUÇÃO}

A deglutição é o processo pelo qual o alimento é preparado e direcionado da boca até o estômago ${ }^{1,2}$. É dividida, basicamente, em três fases: a fase oral (preparatória e propulsiva), consciente e voluntária;

(1) Fonoaudióloga clínica; Mestranda do Curso de Pós-Graduação em Neuropsiquiatria e Ciências do Comportamento da Universidade Federal de Pernambuco.

(2) Professora Adjunta do Departamento de Anatomia da Universidade Federal de Pernambuco; Doutora em Neuropsiquiatria e Ciências do Comportamento da Universidade Federal de Pernambuco.

(3) Terapeuta Ocupacional; Mestranda do Curso de Pós-Graduação em Neuropsiquiatria e Ciências do Comportamento da Universidade Federal de Pernambuco.

(4) Professor Adjunto do Departamento de Neuropsiquiatria e Ciências do Comportamento da Universidade Federal de Pernambuco; Doutor em Neurologia / Neurociências pela Universidade Federal de São Paulo.

Conflito de interesses: inexistente a fase faríngea, consciente e involuntária, ambas comandadas pelo sistema nervoso central; e a fase esofágica, inconsciente e involuntária controlada pelo sistema nervoso somático e autônomo ${ }^{3}$.

A eletromiografia de superfície (EMGs), por se tratar de um exame de caráter não-invasivo e não apresentar contra-indicações surge como uma alternativa para, associada aos exames tradicionais, auxiliar as avaliações clínicas da deglutição fornecendo feedback mais rápido ao paciente e familiares ${ }^{4-6}$.

O registro eletromiográfico é obtido a partir da captação, através de eletrodos de superfície, de um sinal bioelétrico gerado a partir do ponto de inervação do músculo. Este sinalse propaga em direções opostas até atingir as regiões tendíneas ${ }^{7}$, fornecendo informações eletrofisiológicas como duração, amplitude e morfologia do eletromiograma durante uma contração muscular8-10. 
Uma das pesquisas pioneiras no estudo eletrofisiológico da deglutição foi desenvolvida por Doty e Bosma (1956) ${ }^{11}$, entretanto pouco se sabe atualmente sobre o comportamento eletrofisiológico considerado normal.

Desta forma, o objetivo dessa revisão sistemática foi investigar se a literatura aponta valores referenciais para a duração, amplitude e morfologia do eletromiograma durante a deglutição dos seguintes músculos: orbicular da boca, masseter, músculos da região supra-hióidea e músculos da região infrahióidea, cobertos pelo músculo platisma durante a deglutição.

\section{MÉTODO}

A pesquisa foi desenvolvida por três pesquisadores. Dois pesquisadores (LB e MGWS) buscaram os dados de forma independente e cega, inicialmente. O terceiro pesquisador (OGL), instituído como revisor, foi consultado nos casos de dúvida para estabelecer uma concordância entre as idéias.

Foram incluídos, os artigos com seres humanos, de ambos os sexos, cuja amostra estava constituída por sujeitos adultos, idosos saudáveis e sem sequelas neurológicas.

Foram excluídos artigos experimentais e analíticos que abordavam estudos de caso clínico, estudos comparativos de sujeitos com patologias diversas com sujeitos considerados normais.

A busca foi realizada no período entre Março e Abril de 2010. Não houve restrição quanto ao idioma ou ano da publicação. Os descritores foram escolhidos de acordo com as listas DeCS e MeSH. Pela lista do DeCS o descritor foi: Deglutição. Pela lista do MeSH os descritores foram: Deglutition e Swallowing.

Outros descritores não catalogados nas referidas listas foram utilizados para ampliar a busca (Figura 1). As referências dos artigos selecionados foram analisadas para verificar outros estudos que pudessem ter sido omitidos na busca eletrônica.

Foram utilizados os bancos de dados do portal da Bireme (Medline, Lilacs, Ibecs, Scielo, Biblioteca Cochrane, entre outros bancos desse portal), do Pubmed e banco de teses da Capes. A estratégia de busca aplicada seguiu recomendações de Castro, et $a^{12}$, Dickersin, et $a^{1 / 3}$ e a Cochrane Collaboration.

\section{1. (surface electromyography or sEMG or surface EMG)}

\section{2. (deglutition or swallow or swallowing)}

3. (normal)

4. \#1 and \#2 and \#3

Figura 1 - Descritores usados para a busca. Em negrito os descritores da lista DeCS/MeSH

Foram encontrados 28 artigos no portal Pubmed e 33 artigos no portal Bireme potencialmente relevantes e que foram armazenados para análise. Durante a apreciação dos 61 artigos foi identificado que, todos os 28 artigos selecionados do portal Pubmed estavam repetidos no resultado da pesquisa realizada no portal Bireme, ficando 33 artigos para análise. Entre os 33 artigos analisados, 28 foram excluídos por não atenderem aos critérios de inclusão, finalizando a coleta com a inclusão de 5 artigos: Vaiman, 2004 (A) 14; Vaiman, 2004 (B) ${ }^{15}$; Vaiman, 2004 (C) ${ }^{16}$; Vaiman, 2004 (D) ${ }^{17}$; Vaiman, $2005^{18}$.
Após leitura criteriosa e aplicação da escala de Jadad para avaliar a qualidade dos artigos, foi observado que nenhum deles definiu seus estudos como de natureza randomizada e desta forma deixaram de pontuar dois itens dessa escala; todos os artigos fizeram comparações, mas apenas 2 (40\%), Vaiman et al, 2004 (C) e Vaiman et al, 2005, demonstraram comparações adequadas; apenas 1 (20\%) Vaiman et al, 2005 descreveu as perdas e exclusões da amostra. E apenas 1(20\%), Vaiman et al, 2005 foi considerado com boa qualidade segundo a escala de Jadad ${ }^{19}$ (Tabela 1). 
Tabela 1 - Com as pontuações atingidas pelos artigos seguindo os critérios da escala de Jadad

\begin{tabular}{|c|c|c|c|c|c|}
\hline \multirow{2}{*}{ Perguntas } & \multicolumn{5}{|c|}{ Pontuação } \\
\hline & Vaiman (A) & Vaiman (B) & Vaiman (C) & Vaiman (D) & Vaiman, 2005 \\
\hline $\begin{array}{l}\text { 1. O estudo foi descrito } \\
\text { como randomizado? }\end{array}$ & 0 & 0 & 0 & 0 & 0 \\
\hline $\begin{array}{l}\text { 2. A randomização foi } \\
\text { descrita e é } \\
\text { adequada? }\end{array}$ & 0 & 0 & 0 & 0 & 0 \\
\hline $\begin{array}{l}\text { 3. Houve comparações } \\
\text { e resultados? }\end{array}$ & 1 & 1 & 1 & 1 & 1 \\
\hline $\begin{array}{l}\text { 4. As comparações e } \\
\text { resultados foram } \\
\text { descritos e são } \\
\text { adequados? }\end{array}$ & 0 & 0 & 1 & 0 & 1 \\
\hline $\begin{array}{l}\text { 5. Foram descritas as } \\
\text { perdas e exclusões? }\end{array}$ & 0 & 0 & 0 & 0 & 1 \\
\hline Total & 1 & 1 & 2 & 1 & 3 \\
\hline
\end{tabular}

Cada resposta positiva gera 1 ponto na escala, que resulta na avaliação de 0-5 pontos.

\section{REVISÃO DA LITERATURA}

Os artigos do Vaiman, et al 2004 (A, B, C, D) e Vaiman, 2005 apresentam características metodológicas em comum. Todos esses artigos avaliaram ambos os sexos, separaram a amostra em grupos de faixas etárias, utilizaram o mesmo desenho de estudo, e realizaram provas de deglutição em comum e todos visaram estabelecer ou estudar dados eletrofisiológicos de normalidade de músculos envolvidos na deglutição (Tabela 2).

Tabela 2 - Características metodológicas dos artigos

\begin{tabular}{|c|c|c|c|c|c|}
\hline & Vaiman (A) & Vaiman (B) & Vaiman (C) & Vaiman (D) & Vaiman, 2005 \\
\hline $\begin{array}{l}\text { Pop/ } \\
\text { idade }\end{array}$ & $\begin{array}{c}230 \mathrm{M} \mathrm{e} 210 \mathrm{H} / \\
18 \text { a } 78 \text { anos }\end{array}$ & $\begin{array}{c}220 \mathrm{M} \mathrm{e} 200 \mathrm{H} / \\
18 \text { a } 78 \text { anos }\end{array}$ & $\begin{array}{c}230 \mathrm{M} \mathrm{e} 210 \mathrm{H} / \\
18 \text { a } 78 \text { anos }\end{array}$ & $\begin{array}{c}170 \mathrm{M} \mathrm{e} 130 \mathrm{H} / \\
18 \text { a } 81 \text { anos }\end{array}$ & $\begin{array}{c}\text { Do total de } 668 \\
\text { ficaram } 220 \mathrm{M} \mathrm{e} \\
200 \mathrm{H} / 18-79 \\
\text { anos. }\end{array}$ \\
\hline$\overline{D E}$ & POSN & POSN & POSN & POSN & POSN \\
\hline Provas & $\begin{array}{c}\text { DS; DN; D20/17; } \\
\text { CC100; DS1h }\end{array}$ & $\begin{array}{c}\text { DS; DN; D20/17; } \\
\text { CC100 }\end{array}$ & $\begin{array}{c}\text { DS; DN; D20/17; } \\
\text { CC100 }\end{array}$ & DS; DN; D20/17 & CC100 \\
\hline Objetivo & $\begin{array}{c}\text { Estabelecer } \\
\text { dados } \\
\text { normativos para } \\
\text { a duração }\end{array}$ & $\begin{array}{c}\text { Estabelecer } \\
\text { dados } \\
\text { normativos para } \\
\text { a amplitude }\end{array}$ & $\begin{array}{c}\text { Descrever e } \\
\text { avaliar os } \\
\text { diferentes } \\
\text { gráficos de } \\
\text { deglutições ditas } \\
\text { normais }\end{array}$ & $\begin{array}{c}\text { Estimar os } \\
\text { valores de } \\
\text { normalidade dos } \\
\text { registros } \mathrm{e} \\
\text { avaliar os } \\
\text { estágios da } \\
\text { deglutição } \\
\text { normal }\end{array}$ & $\begin{array}{c}\text { Descrever e } \\
\text { estabelecer } \\
\text { valores de } \\
\text { normalidade do } \\
\text { consumo } \\
\text { contínuo de água }\end{array}$ \\
\hline
\end{tabular}

M: mulheres; H: homens; DE: desenho do estudo; POSN: prospectivo observacional de sujeitos normais; DS: deglutição seca; D20/17: deglutição de um volume excessivo de 20 ou $17 \mathrm{ml}$; CC 100: consumo contínuo de $100 \mathrm{ml}$ de água.

As provas realizadas foram: deglutição seca, deglutição normal, deglutição de um volume excessivo de 20 ou $17 \mathrm{ml}$. A prova de deglutição seca consistiu da realização de três deglutições de saliva. A prova de deglutição normal foi realizada após o cálculo do volume médio sorvido de um 
copo de água pelos sujeitos separados por faixas etárias e assim, os grupos 1 e 2 (18-40 anos) deglutiram um volume de $16,5 \mathrm{ml}$; o grupo 3 e 4 (41- 60 anos) deglutiram um volume de $15,5 \mathrm{ml}$; o grupo 5 (41- 60 anos) deglutiu um volume de $13,5 \mathrm{ml}$ e o grupo 6 (idade acima de 70 anos) deglutiu $12 \mathrm{ml}$ em uma única deglutição.

A prova de deglutição de um volume excessivo foi realizada pela oferta do volume de $20 \mathrm{ml}$ de água para os mais jovens que 70 anos e $17 \mathrm{ml}$ de água para os sujeitos com idade superior a 70 anos. O comando foi para deglutirem em um só gole (Tabela 2).

$\mathrm{Na}$ prova de deglutição de saliva monitorada por uma hora, os sujeitos foram orientados a se manterem sentados de forma casual lendo um livro ou revista, enquanto o equipamento de EMGs registrava as deglutições voluntárias de saliva.

Os artigos que realizaram a prova de deglutição normal e a prova de deglutição de um volume excessivo utilizaram volumes diferentes para comparar os valores de amplitude, duração e morfologia do eletromiograma entre os grupos de faixas etárias.

A deglutição de diferentes volumes pode influenciar os resultados eletrofisiológicos obtidos na
$E M^{20-22}$ e gerar dúvidas em relação aos resultados obtidos, ou seja, os resultados encontrados referem-se às diferenças existentes entre os jovens e idosos ou aos diferentes volumes oferecidos? Ou ambos? Por esta razão, os artigos que realizaram essas provas não pontuaram no item que se refere à qualidade das comparações da escala de Jadad (Tabela 1).

Em relação às especificações técnicas do eletromiógrafo, apenas Vaiman (D) e Vaiman, 2005 definiram a frequência de amostragem e ambos utilizaram o valor de $100 \mathrm{~Hz}$. De uma forma geral, os parâmetros utilizados pelos artigos para analisar a amplitude da EMGs foram: média, média bruta, média real e o range.

Nesses artigos não ficou claro como foi feito o processamento quantitativo do sinal. Os grupos musculares em comum avaliados foram: músculo orbicular da boca (MOB) músculo masseter (MM), músculos supra-hióideos (MSH) e os músculos infra-hióideos $(\mathrm{MIH})$, entretanto o músculo orbicular da boca foi excluído das análises por ter demonstrado uma grande variabilidade nos resultados (Tabela 3).

Tabela 3 - Especificações técnicas do eletromiógrafo

\begin{tabular}{|c|c|c|c|c|c|}
\hline & Vaiman $(A)$ & Vaiman (B) & Vaiman (C) & Vaiman (D) & Vaiman, 2005 \\
\hline Músculos & $\begin{array}{l}\mathrm{MOB} ; \mathrm{MM} ; \\
\mathrm{MSH} ; \mathrm{MIH}\end{array}$ & $\begin{array}{l}\text { MOB;MM; } \\
\text { MSH;MIH }\end{array}$ & $\begin{array}{l}\text { MOB;MM; } \\
\text { MSH;MIH }\end{array}$ & $\begin{array}{l}\text { MOB;MM; } \\
\text { MSH; ML }\end{array}$ & $\begin{array}{l}\text { MOB;MM; } \\
\text { MSH;MIH }\end{array}$ \\
\hline$\overline{\text { ETA }}$ & $\begin{array}{c}\text {-Passa-banda } \\
\text { (RMS): } \\
25 \text { a } 450 \mathrm{~Hz} \\
\text {-Filtro notch: } \\
60-\mathrm{Hz} .\end{array}$ & $\begin{array}{c}\text {-Passa-banda } \\
\text { (RMS): } \\
25 \text { a } 450 \mathrm{~Hz} \\
\text {-Filtro notch: } \\
60-\mathrm{Hz} .\end{array}$ & $\begin{array}{c}\text {-Passa-banda } \\
\text { (RMS): } \\
25 \text { a } 450 \mathrm{~Hz} ; \\
\text {-Filtro notch: } \\
\text { 60-Hz. }\end{array}$ & $\begin{array}{c}\text {-Passa-banda: } \\
\text { (RMS): } \\
25 \text { a } 450 \mathrm{~Hz} \\
\text {-Filtro notch: } \\
60 \mathrm{~Hz} \\
\text {-FA de } 100 \mathrm{~Hz}\end{array}$ & $\begin{array}{c}\text {-Passa-banda } \\
\text { (RMS): } \\
25 \text { a } 450 \mathrm{~Hz} \\
\text {-Filtro notch: } \\
60 \mathrm{~Hz} \\
\text {-FA de } 100 \mathrm{~Hz}\end{array}$ \\
\hline Sinal & $\begin{array}{c}\text { Retificado } \\
\text { filtrado }\end{array}$ & $\begin{array}{c}\text { Retificado } \\
\text { filtrado }\end{array}$ & $\begin{array}{l}\text { Retificado } \\
\text { filtrado. }\end{array}$ & $\begin{array}{c}\text { Retificado } \\
\text { Filtrado }\end{array}$ & $\begin{array}{c}\text { Retificado } \\
\text { filtrado }\end{array}$ \\
\hline Parâmetros & Média da duração & $\begin{array}{c}\text { Média da } \\
\text { amplitude e da } \\
\text { duração }\end{array}$ & Eletromiogramas & $\begin{array}{c}\text { Média da } \\
\text { amplitude } \\
\text { e da duração. }\end{array}$ & $\begin{array}{l}\text { Média bruta, a } \\
\text { média real da } \\
\text { amplitude e } \\
\text { média da duração }\end{array}$ \\
\hline
\end{tabular}

MOB: músculo orbicular da boca; MM: músculo masseter; MSH: músculos da região supra-hióidea; MIH = músculos da região infrahióidea; ML= músculos laríngeos; ETA= especificações técnicas do aparelho.

Vaiman (D) afirmou ter estabelecido valores de normalidade para a duração e amplitude da EMGs durante a deglutição, porém apenas Vaiman (A) e Vaiman (B) especificaram esses valores referenciais em tabelas.

Vamain (A) definiu dados referenciais de duração nas provas de deglutição seca, deglutição normal e deglutição de um volume excessivo. Esses dados foram obtidos após comparações entre homens e mulheres e entre faixas etárias e encontram-se na tabela 4.

Embora o artigo do Vaiman, 2005 em sua discussão tenha afirmado ter definido valores referenciais de duração e amplitude para a prova do 
consumo contínuo de $100 \mathrm{ml}$ de água, não fica claro nas suas tabelas de resultados se os valores descritos remetem à dados referenciais.

Desta forma, estão expostos na tabela 5 apenas os valores referencias para a duração da EMGs propostos por Vaiman (A) para a prova de consumo contínuo de $100 \mathrm{ml}$ de água.

Tabela 4 - Valores referenciais para a duração (segundos) obtida pela EMGs, retirados dos artigos do Vaiman (A)

\begin{tabular}{lcc}
\hline & \multicolumn{2}{c}{ Vaiman (A) } \\
& $\mathbf{1 8 - 7 0}$ & $\geq \mathbf{7 0}$ \\
\hline DS & 3,22 & 3,84 \\
DN & 3,34 & 4,50 \\
D20/17 & 4,01 & 4,55 \\
1D CC100 & 1,55 & 1,75 \\
\hline
\end{tabular}

DS- deglutição de saliva; DN- deglutição normal; D20/17 deglutição excessiva de 20 ou $17 \mathrm{ml}$ de água; 1D CC 100 - uma deglutição do consumo contínuo de $100 \mathrm{ml}$ de água.

Tabela 5 - Valores referenciais, propostos pelo artigo do Vaiman, A, para a duração (segundos) na prova de consumo contínuo de $100 \mathrm{ml}$ de água

\begin{tabular}{lccc}
\hline \multicolumn{3}{c}{ Vaiman (A) } \\
\hline Idades & $18-60$ & $61-70$ & 70 \\
CC100 & 10,8 & 13,4 & 17,7 \\
\hline
\end{tabular}

CC100 - consumo contínuo de $100 \mathrm{ml}$ de água.
Vaiman (B) foi o único artigo que, a partir das comparações entre os sexos e entre as faixas etárias, expôs em tabelas valores referencias para a amplitude da EMGs do músculo masseter e dos músculos supra-hióideos nas provas de deglutição seca; deglutição normal; deglutição de um volume excessivo (Tabela 6); e para o consumo contínuo de $100 \mathrm{ml}$ de água (Tabela 7).

Nenhum dos artigos encontrou diferenças significativas entre os sexos, porém nas comparações entre as faixas etárias, os idosos com idade superior a 70 anos demonstraram duração mais prolongada (Vaiman A, D e Vaiman 2005) e amplitude dos MSH mais reduzida (Vaiman B, D e Vaiman 2005) que os mais jovens.

Em relação à morfologia do eletromiograma, Vaiman (C) e Vaiman (D) analisaram o sinal retificado e filtrado por um passa-baixa. Vaiman (C) encontrou que $318(72,27 \%)$ sujeitos demonstraram um gráfico com um único pico, que chamaram de single-share; $50(11,36 \%)$ demonstraram duplo pico (double-share); 23 (5,23\%) demonstraram três picos (triple-share); 49 (11.14\%) sujeitos restantes demonstraram os três padrões de forma mista.

Vaiman $D$ encontrou padrões semelhantes ao estudo do Vaiman (C). Esse estudo encontrou que $217(72,33 \%)$ sujeitos demonstraram um gráfico com um único pico, que chamaram de single-peak, e 33 sujeitos $(11 \%)$ demonstraram duplo pico (doblepeak); e 12 sujeitos (4\%) demonstraram três picos (triple-peak). Os $38(12,66 \%)$ sujeitos restantes demonstraram os três padrões de forma mista.

Tabela 6 - Valores referenciais para a amplitude da EMGs em $\mu \mathrm{V}$ do masseter e músculos da região supra-hióidea durante os vários testes realizados por Vaiman (B)

\begin{tabular}{lccc}
\hline Provas & \multicolumn{3}{c}{ Idade: Range } \\
\hline DS & & & \\
Range MM & $18-30: 4,5-15,9$ & $31-70: 5,54-12,1$ & $70+: 2,94-22,42$ \\
Range MSH & $18-30: 13,4-15,9$ & $31-70: 9,52-49,5$ & $70+: 10,2-42,32$ \\
\hline DN & $18-60: 2,2-31,0$ & $61-70: 1,97-27,69$ & $70+: 3,77-20,0$ \\
Range MM & $18-30: 11,4-63,41$ & $31-50: 12,58-51,6$ & $51-70+: 7,4-44,8$ \\
Range MSH & $18-40: 1,5-37,0$ & $41-70: 1,2-29,4$ & $70+: 4,65-21,13$ \\
\hline D20 ml & $18-30: 19,28-50,80$ & $31-70+: 12,1-47,44$ & \\
Range MM & Range MSH & & \\
\hline
\end{tabular}

Range - intervalo; DS- deglutição de saliva; DN- deglutição normal; D20 - deglutição excessiva de $20 \mathrm{ml}$ de água; 1D 100ml - uma deglutição de 100 ml. MM: masseter; MSH: músculos da região supra-hióidea. 
Tabela 7 - Valores referenciais, propostos pelo artigo do Vaiman, B, para a amplitude (microvolts) na prova de consumo contínuo de $100 \mathrm{ml}$ de água

\begin{tabular}{lcc}
\hline & \multicolumn{2}{c}{ Vaiman (B) } \\
\hline & \multicolumn{2}{c}{ Amplitude do MM } \\
Idades (anos) & $18-70$ & $70+$ \\
CC100 & $0,8-6,2$ & $1,0-7,84$ \\
\hline \multicolumn{3}{c}{ Amplitude do $\mathrm{MSH}$} \\
Idades (anos) & $18-60$ & $61-70+$ \\
CC100 & $3,5-11,5$ & $4,25-16,25$ \\
\hline
\end{tabular}

CC 100 - consumo contínuo de $100 \mathrm{ml}$ de água.

Discutindo sobre a técnica utilizada nos artigos, foi observado que apenas os artigos do Vaiman (D) e Vaiman, 2005 fizeram referência à frequência de amostragem utilizada e ambos referiram o mesmo valor: $100 \mathrm{~Hz}$.

A literatura recomenda que a frequência de amostragem seja de pelo menos $1000 \mathrm{~Hz}$ tendo em vista que os sinais de EMGs para avaliação eletrofisiológica muscular podem ter componentes de frequência de até $300 \mathrm{~Hz}$ a $500 \mathrm{~Hz}$. A definição dessa freqüência deve seguir a regra da taxa de amostragem de Nyquest.

Segundo essa regra, a freqüência de amostragem deve ser de pelo menos o dobro do valor da maior freqüência estimada para a atividade muscular 7 . Quando a taxa de amostragem é menor que a taxa de Nyquest é provável que o sinal captado sofra distorções (aliasing) ${ }^{23,24}$, influenciando nos valores de amplitude e no eletromiograma obtido.

De uma forma geral, os artigos analisaram: a média, média bruta, média real e o range. Porém, não ficou claro como foi feito o processamento quantitativo do sinal para obter esses valores. 0 processamento pode ser feito de uma dessas três maneiras: pico-a-pico, média integrada e root mean square (RMS), sendo este último o mais recomendado. Seu cálculo é feito pela seguinte equação ${ }^{25,26}$.

$$
x_{\text {rms }}=\sqrt{\frac{1}{n} \sum_{i=1}^{n} x_{i}^{2}}=\sqrt{\frac{x_{1}^{2}+x_{2}^{2}+\cdots+x_{n}^{2}}{n}}
$$

Os valores referenciais para a amplitude dos músculos masseter e músculos supra-hióideos durante a deglutição foram propostos no artigo do Vaiman (B). Nesse artigo os autores não especificaram a freqüência de amostragem e concluíram que esses dados podem ser usados para a determinação de um diagnóstico; objetivação das queixas; localização do processo patológico; para comparações dos estágios no pré e pós-operatório e para o monitoramento dos pacientes.

Porém, o sinal eletromiográfico de superfície pode ser influenciado por diversos fatores, que são distintos de sujeito a sujeito como: espessura de tecido adiposo, duração do repouso muscular, velocidade de contração, massa muscular, predomínio de tipo de fibra, mudanças sutis na postura, distância inter-eletrodo e impedância da pele $25-27$. Estes fatores podem causar uma grande variabilidade inter e entre sujeitos.

Tendo em vista todos esses fatores, O'Kane et al, $2010^{28}$ e o próprio Vaiman e Eviatar (2009) ${ }^{8}$ em um artigo de revisão, reconhecem a fragilidade do método. Vaiman e Eviatar, 2009 afirmaram que os valores de referência propostos são úteis para a realização de triagem da função de deglutição e auxílio das avaliações clínicas, mas não oferecem informações suficientes para o estabelecimento de diagnóstico como acreditado anteriormente.

Tendo em vista esta grande variabilidade a literatura propõe a realização de técnicas de normalização do sinal, como: contração voluntária máxima, contração voluntária submáxima, valores de contração submáxima para o pico de contração, avaliação percentual pela linha de base, entre outras $^{8,25,26 .}$.

\section{CONCLUSÃO}

Os valores referenciais propostos pelos artigos estudados podem não ser replicáveis, pois Vaiman (A), Vaiman (B) e Vaiman (C) não especificaram o valor da freqüência de amostragem e realizaram provas de deglutição sem padronização dos volumes. Vaiman (D) e Vaiman, 2005 especificaram uma frequência de amostragem que não seguiu a regra de amostragem de Nyquest, potencializando a obtenção de dados distorcidos da atividade muscular. Tendo em vista a variabilidade inter e entre sujeitos, a literatura sugere a realização de técnicas de normalização do sinal eletromiográfico. 


\begin{abstract}
Background: normal surface electromyography (sEMG) of muscles involved in swallowing. Purpose: to investigate if the literature indicates normal parameters for duration, amplitude and characteristics of the electromyograms of the muscles involved in swallowing (orbicularis oris, masseter, muscles of the suprahyoid muscles and the infra-hyoid region covered by the platysma muscle). Conclusion: the search resulted in 33 references, of which only five were within the inclusion criteria. Only one reference was classified with good level of quality by the Jadad scale with modifications. The selected articles cannot point reliable reference values mainly for EMG amplitude and morphology, because they used a inappropriate sampling rate for the EMG recordings that maximizes the achievement of distorted data for the muscle activity. Literature suggests the normalization of techniques for the electromyographic signal due to the variability between and among subjects.
\end{abstract}

KEYWORDS: Electromyography; Deglutition; Muscles

\section{REFERÊNCIAS}

1. Schindler JS, Kelly JH. Swallowing disorders in the elderly. The Laryngoscope. 2002; 112: 589-602. 2. Logemann JA, Evaluation and Treatment of Swallowing Disorders. Austin, TX: Pro-ed; 1998.

3. Manrique D. Avaliação Otorrinolaringológica da Deglutição. In: Furkin AM, Santine CS (org). Disfagias orofaríngeas. São Paulo: Pró-Fono; 2004. 4. Crary MA, Baldwin BO. Surface electromyographic characteristics of swallowing in dysphagia secondary to brainstem stroke. Dysphagia. 1997; 12:180-7.

5. Logemann JA, Evaluation and Treatment of Swallowing Disorders. Austin, TX: Pro-ed, 1998.

6. Ertekin C, Palmer JB. Physiology and electromyography of swallowing and its disorders. In: AMBLER Z et al. Clinical neurophysiology at the beginning of the 21 st Century supplements to $\mathrm{J}$ Clin Neurophysiol. 2000; 53: 148-54.

7. Portney L, Roy SH. Eletromiografia e testes de velocidade de condução nervosa. In: O`Sullivan SB, Schmitz TJ. Fisioterapia: avaliação e tratamento. São Paulo: Manole, 2004.

8. Vaiman M, Eviatar E: Surface electromyography as a screening method for evaluation of dysphagia and odynophagia. Head \& Face Medicine. 2009; 5(9): 1-11.

9. Coriolano, MGWS, Lins, OG, BELO, LR, Carneiro D, Moraes SRA, Amdore AG, Oliveira, JAL, Silva DM. Monitorando a deglutição através da eletromiografia de superfície. Revista CEFAC. 2010; 12 (3).

10. Vaiman M. Standardization of surface electromyography utilized to evaluate patients with dysphagia. Head \& Face Medicine. 2007; 3:26.

11. Thexton J, Crompton A. W: Electromyographic activity during the reflex pharyngeal swallow in the pig: Doty and Bosma (1956) revisited. J Appl Physiol. 2007; 102: 587-600.

12. Castro AA, Clarck OAC, Atallah $A N$ et al. Optimal search strategy for clinical trials in the Latin American and Caribbean Health Science Literature Database (LILACS database): Update. Med J/Rev Paul Medm. 1999;117(3): 138-9.

13. Dickersin K, Scherer R, Lefebvre $C$ et al. Identifying relevant studies for systematic reviews Systematic Reviews. BMJ. 1994; 309:1286-91.

14. Vaiman M, Eviatar E, Segal S. Surface electromyographic studies of swallowing in normal subjects: A review of 440 adults. Report 1. Quantitative data: Timing measures. Otolaryngology-Head and neck Surgery. 2004; 131(4):548-55 (A).

15. Vaiman M, Eviatar E, Segal S. Surface electromyographic studies of swallowing in normal subjects: A review of 440 adults. Report 2. Quantitative data: Amplitude measures. Otolaryngology-Head and neck Surgery. 2004; 131(5):773-80 (B).

16. Vaiman M, Eviatar E, Segal S. Surface electromyographic studies of swallowing in normal subjects: A review of 440 adults. Report 3. Qualitative data. Otolaryngology-Head and neck Surgery. 2004;131(6): 977-85 (C).

17. Vaiman M, Eviatar E, Samuel S. Evaluation of Normal Deglutition with the Help of Rectified SurfaceElectromyography Records. Dysphagia. 2004; 19:125-32 (D).

18. Vaiman M, Gabriel C, Eviatar E, Segal S. Surface electromyography of continuous drinking in healthy adults. The Laryngoscope. 2005; 115: 68-73.

19. Sampaio RF, Mancini MC. Estudos de Revisão Sistemática: Um guia para síntese criteriosa da evidência científica. Rev. Bras. Fisioter. 2007; 11(1): 83-9. 
20. Dantas RO, Dodd: Effects of volume and consistency on swallow-induced submental and infarhioid electromyography activity. Braz J Med Biol Res 1990;23:37-44.

21. Lagerlund TD Volume conduction. In: Daube JR, ed. Clinical Neurophysiology. Philadelphia: FA Davis Co, 1996; 29-39.

22. Belo LR, Lins OG, Cunha DA, Amorim CF, Castro $S$ : Eletromiografia de superfície da musculatura supra-hióidea durante a deglutição de idosos sem doenças neurológicas e idosos com Parkinson. Revista CEFAC 2009;11(2):268-80.

23. Gerleman DG, Cook TM. Instumentation. In: US Department of health and human services. Selected topics in surface electromyography for use in occupational setting. expert perspectives.USA: DHS (NIOSH);1992. p.56.
24. Enoka RM. Bases Neuromusculares da Cinesiologia. São Paulo: Manole, 2000.

25. Cram J, Kasman GS. The Basics of Electromyography. In: Criswell E. Cram's Introduction to Surface Electromyography. $2^{\mathrm{a}}$ edição. Massachusets: Jones and Bartlett Publishers; 2011. p.35-61.

26. Portney L. Eletromiografia e testes de velocidade de condução nervosa. In: O'Sullivan SB, Schmitz TJ. Fisioterapia: Avaliação e Tratamento. São Paulo: Manole; 1993. p. 183-217.

27. DeLucaCJ.TheUse of SurfaceElectromyography in Biomechanics. Journal of applied biomechanics. 1997; 13:135-63.

28. O'Kane L; Groher ME, Silva K, Osborn L. Normal Muscular Activity During Swallowing as Measured by Surface Electromyography. Annals of Otology, Rhinology \& Laryngology 2010; 119(6):398-401.

http://dx.doi.org/10.1590/S1516-18462011005000072

RECEBIDO EM: 07/12/2010

ACEITO EM: 31/03/2011

Endereço para correspondência:

Luciana Rodrigues Belo

Rua Abel de Sá Bezerra Cavalvanti. 161, apt 601,

Casa Amarela

Recife - PE

CEP: 52051-270

E-mail: lucianabelo@yahoo.com.br 